\section{Potassium Iodide in Bronchial Asthma}

SIR,-Last year two cases of swelling of the salivary glands after oral potassium iodide were described in the B.M.f. ${ }^{1}$ These and other unwanted effects may have brought this substance into disrepute, which Strümpell' regarded as one of the best remedies for bronchial asthma. On the other hand Alstead ${ }^{3}$ found that low doses of potassium iodide were ineffective, and this may have contributed to the fact that it was little used. I have investigated the desired and the unwanted effects of potassium iodide more closely in a large number of patients.

I have evaluated the records of 2,404 patients treated with potassium iodide in the Asthma Clinic of the Free University of West Berlin during the last 10 years for periods between three weeks and 10 years. All patients $(1,184 \mathrm{men}, 1,120$ women, and 100 children) suffered from chronic obstructive disease of the airways such as bronchial asthma or chronic bronchitis. The dosage of potassium iodide varied from 3.6 to $36 \mathrm{~g}$. daily. As a blind trial was not possible because of the characteristic taste and aftertaste of the drug, it was given for four consecutive days, followed by a free interval of three days ${ }^{5}$ and continued in the same rhythm. In the normal course of this treatment phlegm was expectorated more freely and the cough decreased during the four days of potassium iodide administration; this, therefore, also improved breathlessness. During the subsequent free interval these complaints often gradually increased again. Such a course of events could not possibly have been anticipated by a patient, and if it was spontaneously reported by him this was regarded as objective evidence of the potassium iodide effect. During treatment with the iodide the morning cough disappeared or was shortened, sometimes with the lowest, sometimes with higher doses, and the use of antispasmodic aerosols and the exercise dyspnoea decreased. A high percentage of the patients improved considerably, details of which are given elsewhere. ${ }^{4}$

There were unwanted side-effects after 1236 hours and others occurring after weeks or months. The former were swelling of the salivary glands, iodine coryza, and iodine acne (in $7 \%$ of all patients). The swelling of the salivary glands, which sometimes resembled mumps or Quincke's oedema, usually does not recur if after a short interval potassium iodide is given in small and gradually increasing dosage. The long-term side-effects, occurring after some months or years, were mainly stomach upsets and myxoedema. They were dose-dependent. The incidence of stomach complaints increased from $10.9 \%$ (of 1,225 patients) with small doses to $40.7 \%$ (of 103 patients) with the highest dosage ; that of myxoedema from $0.2 \%$ (of 1,225 patients) to $2 \cdot 9 \%$ (of 103 patients). The danger of myxoedema, therefore, appears less than suggested by other observers. $^{67}$ It can be recognized easily and treated successfully. In $3.4 \%$ of the total 2,404 patients the potassium iodide therapy was abandoned because of stomach complaints.

It appears, therefore, that the unwanted effects of potassium iodide are no more important than those of many other remedies, and that its benefits can be considerable, particularly in the patients with viscous phlegm.

These results should encourage more physicians to treat the secretion of too much or too viscous mucus, occurring in bronchia asthma and releated diseases, with potassium iodide.-I am, etc.,

\section{Free University,}

Christine Bernecker.

\section{REFERENCES}

Harden, R. McG., British Medical fournal, 1968

Strümpell, A., Lehrbuch der speciellen Pathologie und Therapie der inneren Krankheiten, 1914, 19 th ed. Leipzig, Vogel.

Alstead, S., Lancet, 1939, $2,932$.

- Bernecker, Ch., Acta allergologica, 1969, in press. Herxheimer, H., Management of Bronchial

Begg, T. B., and Hall, R., Quarterly fournal of
Medicine, 1963, 32, 351.

' Frey, H., Acta Endocrinologica, 1964, 47, 105.

\section{Inappropriate Lactation}

SIR,-Various forms of drug therapy, especially certain preparations extensively used in modern psychiatric practice, mus now be the commonest cause of non-puerperal galactorrhoea, and Drs. N. E. F. Cartlidg and P. Hodgson (30 August, p. 529) have performed a valuable service in drawing attention to this problem. Our experience suggests that cases of this type are being referred in increasing numbers to gynaecological clinics, where failure to appreciate the likelihood of an iatrogenic aetiology may lead to unnecessary time-consuming and expensive investigations, with consequent needless distress and anxiety to the patient.

A number of psychotropic drugs have already been implicated as causing galactorrhoea, ${ }^{1-3}$ and we personally have observed the condition in patients on chlordiazepoxide and thioridazine, but we would like to describe here a case in which spurious lactation was apparently caused or aggravated by a monoamine oxidase inhibitor, phenelzine, which we believe has not previously been implicated.

The patient, an unmarried 19-year-old woman, had a long history of severe personality disorder with phobic and depresssive symptoms. Treatment with thioridazine $50 \mathrm{mg}$. three time a day, and phenelzine $15 \mathrm{mg}$. three times a day, was started in November 1967. Prior to this there had been no history of breast activity. On review four months later her breasts were enlarged and her complaint of a milky discharge from them was readily confirmed. In view of the known association of thioridazine with galactorrhoea this drug was replaced by diazepam $5 \mathrm{mg}$. three times a day. She was seen again three months later, when lactation and breast enlargement were markedly increased, and it transpired that this had followed a selfprescribed doubling of the dosage of phenelzine. Withdrawal of phenelzine was followed by rapid diminution of lactation and breast size despite continued treatment with diazepam. Menstruation and the findings on pelvic examination remained normal throughout these events. $X$-rays of the skull and pituitary fossa, urinary ketosteroid assays, electroencephalography, and C.S.F. investigations revealed no abnormality.

The onset of lactation in this case could be related to thioridazine, but this could not account for the exacerbation which occurred several weeks after its discontinuation. This ncreased lactation occurred after the administration of diazepam (which like phenelzine has not previously been reported to cause galactorrhoea), but the marked resolution of symptoms on stopping phenelzine, despite continued diazepam therapy, makes it appear unlikely that the latter was the cause of the increased breast activity. On balance, therefore, the evidence points to phenelzine as the cause of lactation in this patient, and this conclusion is supported by a personal communication from the manufacturers to one of us (M. S.) describing a similar, unpublished, case occurring in a post-menopausal woman.-We are, etc.,

M. Segal.

R. F. HEYs.

General Hospital,
Halifax, Yorks.

Lampe, W. T., Metabolism, 1967, 16, 257. Sulman, F. G., and Winnik, H. Z., Lancet, 1956 1,161

Hooper, J. H., jun., Welch, V. C., and Shakel-
ford, R. T., fournal of the American Medical Association, 1961, 178, s06.

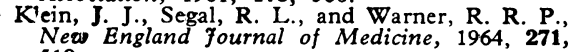

\section{Selective Vagotomy without Drainage}

SIR,-Mr. H. Burge and his co-workers (20 September, p. 690) have made a most helpful contribution in showing that a drain. age procedure is not essential after selective vagotomy in patients who do not have any pyloroduodenal stenosis, but it is possible that they may find similar results in patients with a truncal vagotomy.

I have done barium meal studies using fluid barium over several years on all vagotomy patients seven to ten days after operation, and on many of these three months or more later. Some had selective vagotomy, usually with preservation of the pyloric branch and some had truncal vagotomy. I was also able to compare pyloroplasty with gastrojejunostomy, and in neither type of drainage could I find any difference in emptying between the selective and truncal vagotomy patients. Patients with delay in emptying fell into two groups, those with early and usually short-lived retention, and those developing retention in the second week after operation which persisted for a variable time. In the former group the retention seemed to be due to a temporary inability of the stomach to contract, because when on occasion anxiety led one to do a laparotomy the drainage stoma was usually wide open, and this occurred with both selective and truncal vagotomies. The later speed of emptying seemed to be related to a return of stomach tone together with a degree of peristalsis restricted to the pyloric end, and also on the rate at which the duodenum and/ or upper jejunum accepted barium and propelled it forwards. Here again there was no difference between truncal and selective vagotomies. ${ }^{1}$

It is true that the pylorus is put out of action by the drainage procedure, but if the pyloric branch does have a motor function it is likely that the selective vagotomy patients would have shown peristaltic activity of the pyloric antrum earlier and more often than the truncal vagotomy patients. The preservation of the vagal duodenal innervation which is present in selective vagotomy patients through the hepatic and gastroduodenal plexus irrespective of division of the pyloric branch seems to make no difference in emptying.

The important factor in emptying after selective vagotomy preserving the pyloric branch or truncal vagotomy may not be the 